MARCUS HOLTZ

(Julius-Maximilians-Universität Würzburg, Universitätsarchiv)

\title{
DAS UNIVERSITÄTSARCHIV WÜRZBURG UND SEINE STELLUNG INNERHALB DER BAYERISCHEN UNIVERSITÄTSARCHIVE
}

\section{DiE JULIUS-MAXIMILIANS-UNIVERSITÄT WÜRZBURG UND IHR ARCHIV}

Im Jahr 1402 gründete Fürstbischof Johann von Egloffstein die Universität Würzburg. Es war nach Erfurt, Heidelberg und Köln die vierte derartige Gründung auf dem Gebiet des heutigen Deutschlands. Dieser ersten Würzburger Universität war jedoch nur ein kurzes Leben beschieden, da sich die finanzielle Ausstattung mit der Zeit als unzureichend erwies. Die hohe Schuldenlast, die Egloffsteins Nachfolger Johann von Brunn anhäufte, verbunden mit dessen geringem Interesse an der Universität besiegelten ihr Schicksal. Die letzte überlieferte Nachricht über die Universität stammt aus dem Jahr 1427, danach verschwindet sie im Nebel der Geschichte. Fürstbischof Julius Echter von Mespelbrunn gelang 1582 die Wiederbelebung der Universität, zudem sorgte er für eine ausreichende Ausstattung. Als Ausdruck seiner Ver- 
bundenheit ließ er nach seinem Tode sein Herz in der Universitätskirche beisetzen.

Die Universität besteht nun ununterbrochen seit über 425 Jahre, die Überlieferung ihrer Geschichte weist hingegen einige markante Brüche auf. Neben Aktenverlusten schon in der Frühen Neuzeit war der Bombenangriff vom 16. März 1945 das verheerendste Ereignis. Archivalien aus der Zeit bis 1700 fehlen heute fast vollständig und müssen durch Sammlungsgut substituiert werden. Die besonders fruchtbare Zeit seit dem Beginn des 19. Jahrhunderts, in der nicht allein die Würzburger Physik und Medizin herausragende Forschung leisteten ${ }^{1}$, ist hingegen recht gut überliefert.

Heute studieren an der Julius-Maximilians-Universität über 22000 Studenten in 226 Studiengängen. Dabei werden sie von 366 Professorinnen und Professoren betreut. Mit mehr als 3700 Beschäftigten (ohne Universitätsklinik) gehört die Universität zu den größten Arbeitgebern in der Region.

\section{DAS UNIVERSITÄTSARCHIV}

Die rechtliche Grundlage der bayerischen Universitätsarchive bildet Art. 14 Abs. 1 Satz 1 des Bayerischen Archivgesetzes von

1 So lehrten beispielsweise Carl Caspar von Siebold (1736-1807), Friedrich Wilhelm Schelling (1775-1854), Matthias Lexer (1830-1892), Rudolf Virchow (1821-1902), Felix Dahn (1834-1912), Franz Brentano (1838-1917), Julius Sachs (1832-1897), Theodor Boveri (1862-1915) und Oswald Külpe (1862-1915) hier. Sieben Nobelpreisträger lehrten, vier promovierten oder habilitierten und 3 studierten an der Alma Julia: erhältlich im Internet: http:// www.uni-wuerzburg.de/ueber/universitaet_wuerzburg/persoenlichkeiten/ nobelpreise (besucht am 5. März 2011). 
1989/1999². Die Einrichtung des Würzburger Universitätsarchivs erfolgte 2003-2008. Derzeit ist das Archiv dem Kanzler direkt zugeordnet.

Die Tätigkeit des Universitätsarchivs umfasst sieben Bereiche. Dies sind der Umgang mit zukünftigen Herausforderungen, der Kernbereich Aussonderung / Erschließung / Bestandserhaltung, die Pflege einer Fachbibliothek zur Archivwissenschaft und Universitätsgeschichte, Sammlungen und Nachlässe, die Benutzerbetreuung, Beratung der Verwaltung und der Fakultäten im Bereich Schriftgut sowie die Öffentlichkeitsarbeit. Seit Anfang 2010 wird zudem die Funktion des Sprechers des Arbeitskreises der bayerischen Universitätsarchive durch den Archivleiter wahrgenommen.

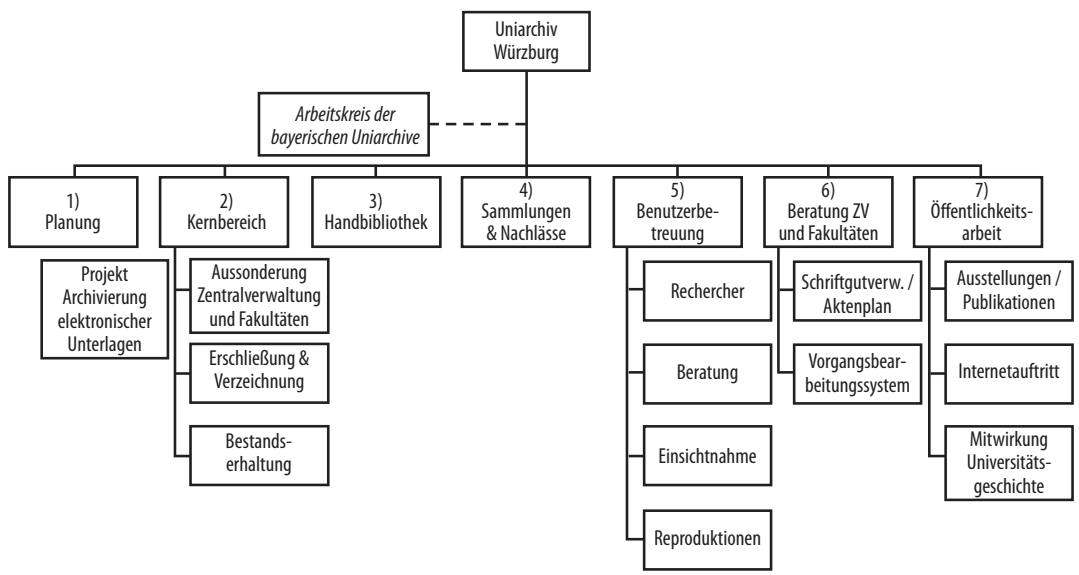

2 Bayerisches Archivgesetz (künftig zitiert als: BayArchivG) vom 22. Dezember 1989, geändert durch Gesetz vom 16. Dezember 1999, erhältlich im Internet: http://www.gda.bayern.de/aufgaben/archivgesetz.php (besucht am 7. März 2011). 


\section{BESTÄNDE}

Das Archivgut umfasst derzeit etwa 1500 laufende Meter. Da das Archiv erst in jüngster Zeit eingerichtet wurde, befinden sich noch umfangreiche Bestände (insbesondere aus der Zeit ab 1946) vor Ort in den einzelnen Fakultäten. Das Archivgut umfasst neben der eigentlichen Überlieferung der Universität auch diejenige der eingegliederten Pädagogischen Hochschule und ihrer Vorgängereinrichtungen (Lehrerbildungsanstalten) sowie Nachlässe und Sammlungsgut. Archivalien des frühneuzeitlichen Stiftungsbesitzes (Rittergüter, aufgelöste Klöster, Grundherrschaft) stellen eine Besonderheit mittelalterlicher und frühneuzeitlicher Universitäten dar.

\section{BAYERISCHE UNIVERSITÄTSARCHIVLANDSCHAFT}

Bayern ist eine alte Universitätslandschaft, mit vier alten, vor dem Ende des Zweiten Weltkriegs gegründeten Universitäten. Die Wurzeln von zwei Universitäten (Ludwig-Maximilian in München und Julius-Maximilian in Würzburg) reichen dabei bis in das Mittelalter zurück.

1472 gründete Herzog Ludwig der Reiche von Bayern-Landshut in Ingolstadt die erste Universität im Herzogtum Bayern. Die militärische Bedrohung während der Napoleonischen Kriege führte im Jahre 1800 zur Verlegung der Universität nach Landshut. Hier fanden während der Regierung König Maximilians I. ${ }^{3}$ umfassende Reformen statt, was auch in der Namensänderung zu Ludwig-Maximilians-Universität Ausdruck findet. Kurz nach

3 Bis 1806 als Max IV. Joseph Kurfürst. 
dem Regierungsantritt König Ludwigs I. verlegte dieser 1826 die Universität nach München.

Die Friedrich-Alexander-Universität Erlangen-Nürnberg wurde 1742 von Markgraf Friedrich von Brandenburg-Bayreuth zunächst in Bayreuth gegründet, aber bereits im folgenden Jahr nach Erlangen verlegt. Besondere Förderung erfuhr die Universität durch Markgraf Karl Alexander von Brandenburg-AnsbachBayreuth. Mit der Eingliederung der Handelshochschule (1961) und der Pädagogischen Hochschule (1972) kam Nürnberg als Standort der Universität hinzu.

Die Technische Universität München wurde 1868 als Polytechnische Schule gegründet und erhielt 1877 die Bezeichnung Technische Hochschule München, 1970 schließlich Technische Universität München. Ein wichtiger Schritt auf dem Weg zur Gleichstellung mit den Universitäten war 1901 die Verleihung des Promotionsrechts. 1930 wurde die Hochschule für Landwirtschaft und Brauerei Weihenstephan der Hochschule eingegliedert.

Die jüngeren bayerischen Universitäten können bei ihrer Entstehung auf ähnliche Entstehungsgeschichten zurückblicken. In der Regel bestand in den katholischen Landesteilen am Ort bereits ein Lyzeum. Alle noch bestehenden Lyzeen wurden 1923 in philosophisch-theologische Hochschulen umgewandelt und diese dann mit der Universitätsgründung den neuen Universitäten, meist als eigene Fakultäten, eingegliedert.

Die 1962 als vierte Landesuniversität gegründete Universität Regensburg geht auf das Lyzeum von 1773 zurück, die 1978 gegründete Universität Passau auf das Lyzeum von 1803.

In Augsburg und Bamberg gab es den historischen Ablauf vom Lyzeum über die philosophisch-theologische Hochschule zur Universitätsgründung ebenfalls. Die Bildungstradition beider Universitäten geht aber über das Lyzeum von 1803 hinaus. 
In Bamberg löste das Lyzeum eine ältere Universität ab. Die Academia Bambergensis von 1647 mit zwei Fakultäten war 1773 von Fürstbischof Adam Friedrich von Seinsheim zur Universität erhoben worden. Diese Universität bestand jedoch nur wenige Jahrzehnte und wurde mit dem Übergang des Fürstbistums Bamberg an das Kurfürstentum Bayern 1803 aufgelöst. Die 1972 aus der Vereinigung der Pädagogischen Hochschule (seit 1958) und der Philosophisch-Theologischen Hochschule entstandene Gesamthochschule Bamberg (seit 1979 Universität) versteht sich als Nachfolgerin dieser ersten Universität, deren Siegel und Namen sie übernommen hat.

Die Universität Augsburg kann mehrere Traditionslinien vorweisen. Mit der Gründung der Universität Augsburg endet nicht nur die Geschichte des Augsburger Lyzeums sondern auch die des Lyzeums im benachbarten Dillingen ${ }^{4}$. Dort war 1551 eine Universität mit nur zwei Fakultäten gegründet worden, die bis zur Säkularisation 1803 bestand und ähnlich wie in Bamberg von einem Lyzeum abgelöst wurde ${ }^{5}$. Eine weitere Traditionslinie hat die Universität Augsburg mit der 1972 eingegliederten pädagogischen Hochschule Augsburg. Die Akten der ehemaligen Pädagogischen Hochschule befinden sich im Augsburger Universitätsarchiv. Die Überlieferungen der eingegliederten pädagogischen Hochschulen werden in den meisten Universitätsarchiven aufbewahrt.

Einen Sonderfall bildet die Universität Bayreuth, die ohne solche Vorläufer geschaffen wurde. Im protestantischen Territorium Brandenburg-Bayreuth war nie ein Lyzeum entstanden und

${ }^{4} \mathrm{Zu}$ Beginn des 16. Jahrhunderts war nach der Reformation in der Reichsstadt Augsburg die Residenz des Fürstbischofs von Augsburg nach Dillingen verlegt worden.

5 Vgl. auch W. Lengger, Das Problem mit der Tradition, oder: Wird die Universität Augsburg wirklich erst 40 Jahre alt?, in: Stätte des Wissens, hg. v. W. Lengger, S. Paulus, W.E.J. Weber, (2010), S. 57-65. 
es hat somit keine philosophisch-theologische Hochschule gegeben. Zudem war die Bayreuther pädagogische Hochschule wie alle anderen 1972 eingegliedert bzw. aufgelöst worden, bestand also zum Zeitpunkt der Universitätsgründung 1975 schon nicht mehr.

Die Universität der Bundeswehr in München wurde 1980/1981 mit der Verleihung des Promotions- und Habilitationsrechts voll anerkannt und lässt seit 2001 auch zivile Studenten zu.

Zur Geschichte der katholischen Universität Eichstätt-Ingolstadt findet sich Näheres im Beitrag von Herrn Prof. Zschaler.

Aufgrund dieser Entstehungsgeschichten lassen sich in den Universitätsarchiven weit über das eigentliche Gründungsjahr der Universität zurückreichende Archivalien der Vorläufereinrichtungen finden.

Gründungsjahre der elf Universitäten in Bayern

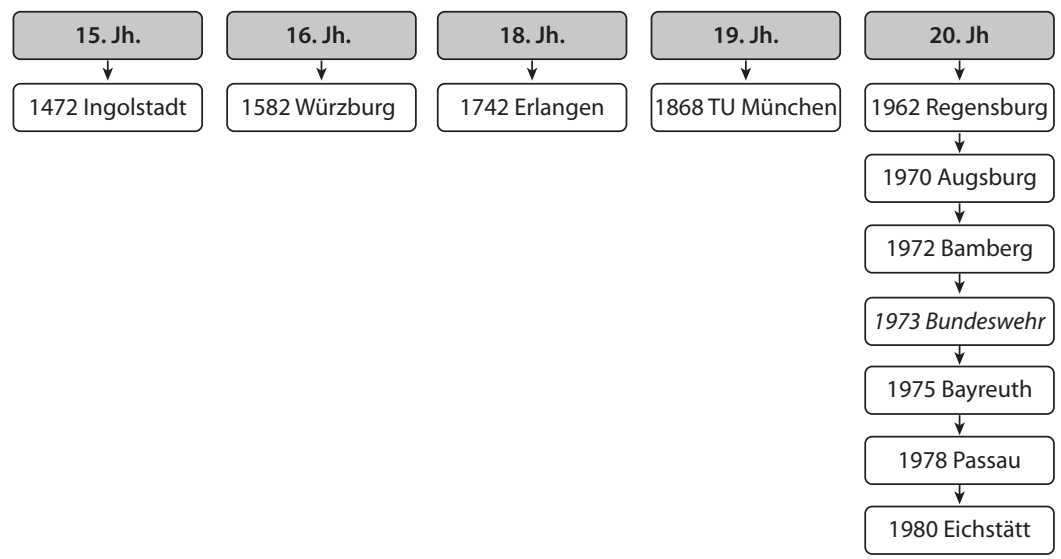

Verbindet man Alter und Größe (i.e. Zahl der Studierenden) zur Charakterisierung der Universitäten, so stellt sich die Frage, wie diese Faktoren sich auf die Universitätsarchive auswirken. 
Eine hohe Studierendenzahl bedeutet viele Lehrstühle und Fachbereiche und eine entsprechend umfangreiche Verwaltung. Für die Archive ergibt sich daraus die ungefähr zu erwartende Menge der jährlich auszusondernden Akten. Das Alter definiert den Umfang der zu pflegenden historischen Archivbestände und eine entsprechend hohe Frequenz an Forschung und Benutzerbetreuung. Alter und Größe sind somit die zwei Faktoren, aus denen sich die Arbeitsbelastung eines Universitätsarchivs wesentlich ableitet.

Studierende im Wintersemester 2010/2011

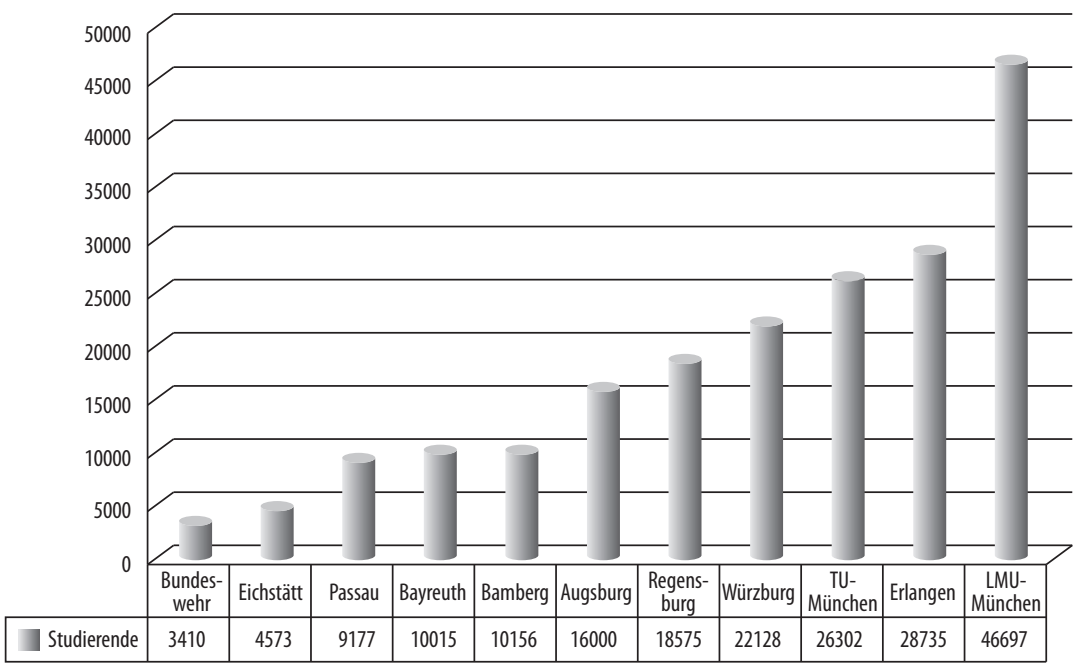

Mit Ausnahme der LMU München haben aber weder Alter noch Größe der Universität eine signifikante Auswirkung auf die Größe oder Ausstattung der Universitätsarchive. 


\section{ATTRAKTIVITÄT AUS TRADITION?}

Eine augenfällige Parallele besteht zwischen dem Alter der Universitäten und den Studentenzahlen. Die vier ältesten Universitäten sind auch diejenigen mit den meisten Studierenden. Wie wichtig historische Tradition als Faktor für die Attraktivität einer Hochschule ist, mag offen bleiben. Sie wird aber als unbezahlbares Alleinstellungsmerkmal im Wettbewerb der Hochschulen einen zunehmenden Stellenwert gewinnen. Dies gilt gerade auch in Abgrenzung zu den Fachhochschulen, die sich immer stärker den Universitäten annähern. In diesem Bereich können die Archive eine erhebliche Außenwirkung erzielen. Die jüngeren Universitäten sind hier mit Blick auf die Tradition von morgen bestens aufgestellt.
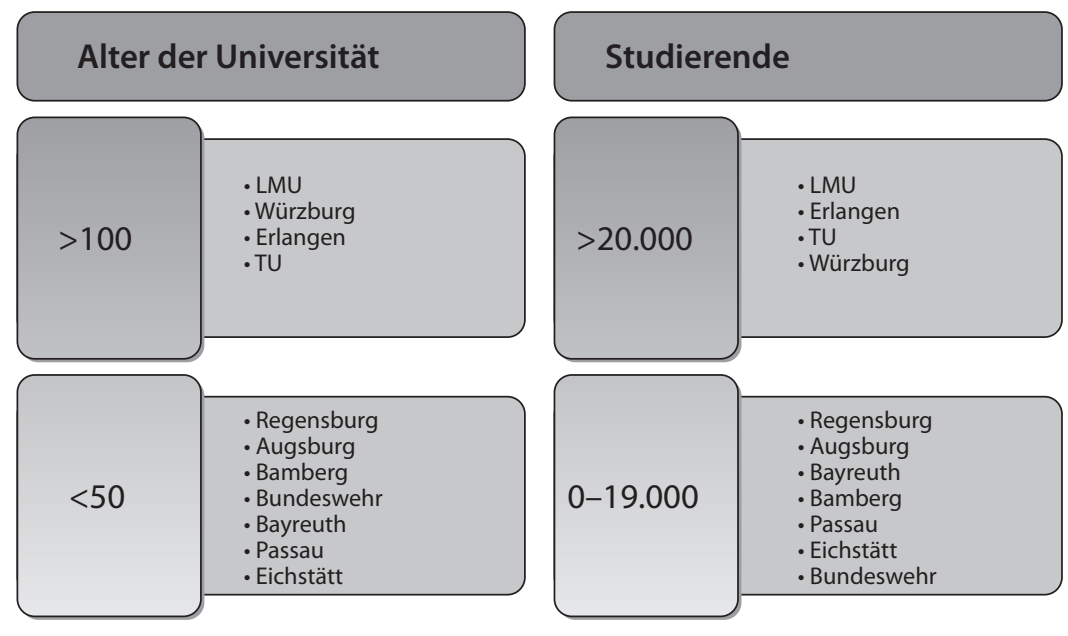


\section{ORGANISATORISCHE VERANKERUNG}

Die organisatorische Verankerung des Archivs in der Universität hat eine nicht unbedeutende Auswirkung auf die Wahrnehmung des Archivs in der Öffentlichkeit und innerhalb der Universität.

Die enge Verzahnung von Verwaltung und Archiv führt zur verbreiteten Angliederung des Archivs an die Zentralverwaltung. Zwischen Archiv und Verwaltung findet ein reger Austausch statt, da Akten nach 10-30 Jahren in das Archiv abgegeben werden. Für die Erledigung langfristiger Aufgaben wird immer wieder auch auf Archivgut zurückgegriffen werden müssen. Umgekehrt findet häufig ein Zugriff des Archivs auf die Registratur statt. Desweiteren ist die Beratung der Verwaltung in allen Fragen der Schriftgutverwaltung (vom Aktenplan bis zur Einführung eines Vorgangsbearbeitungssystems) eine Aufgaben des Archivs.

Eine direkte Anbindung des Archivs an die Hochschulleitung betont zumeist eine besondere historische Tradition der Universität, die sie von jüngeren Universitäten abhebt und die vor allem durch das Archiv repräsentiert wird.

Eine Anbindung des Archivs an die Universitätsbibliothek ergibt sich gelegentlich aus einer gemeinsamen Nutzung der Magazinräume und des Lesesaals. Bibliothek, Museum und Archiv sind zwar verwandte Einrichtungen, haben jedoch signifikant andere Methoden und Aufgaben. Problematisch ist, dass die Wahrnehmung von Bibliothek und Archiv in der Öffentlichkeit häufig verschwimmt, da die meisten Menschen eine Bibliothek oder ein Museum kennen, von einem Archiv aber nur eine diffuse Vorstellung haben. Dies ist kaum verwunderlich, da wichtige Kernaufgaben des Archivs nicht durch Serviceleistungen gegenüber der Öffentlichkeit sondern durch Serviceleistungen gegen- 
über der Verwaltung gekennzeichnet sind. Dieser Unterschied hat spürbare Auswirkungen auf den allgemeinen Kenntnisstand über Archive. Eingebunden in eine Bibliothek besteht die Gefahr, dass das wesentlich kleinere Archiv in der Bibliothek „verschwindet" oder fachfremden Interessen untergeordnet wird. In der Praxis zeigen sich bei dieser Konstruktion erhebliche negative Effekte für die Archive. Sehr positiv ist es daher, dass es Anfang 2010 gelungen ist, das Universitätsarchiv Regensburg aus der Universitätsbibliothek auszugliedern und es in die Zentralverwaltung zu integrieren.

Die wohl ideale Organisationsform ist die zentrale Einrichtung. Dies geschieht nicht primär, weil das Archiv durch das bayerische Archivgesetz eine eigene rechtliche Grundlage außerhalb des Hochschulgesetzes hat. Sie verdeutlicht vielmehr die vielschichtigen Aufgaben für die Gesamtuniversität und dient als deutlich wahrnehmbare Stelle für Nachforschungen zur Geschichte der Universität. Sie kann somit gerade an den älteren Universitäten eine besondere Außenwirkung erzielen. Hierdurch werden Kooperationen mit inner- und außeruniversitären Einrichtungen ebenfalls erleichtert.

$\mathrm{Zu}$ betonen ist an dieser Stelle, dass eine zentrale Einrichtung an den Aufgaben orientiert und somit unabhängig von Größe und Alter ist. Was Personalstärke und Haushaltsmittel angeht, wird ein Universitätsarchiv nie auf einer Stufe mit Einrichtungen wie der Universitätsbibliothek oder dem Rechenzentrum stehen. Dass dies keinen Einfluss auf die Stellung als zentrale Einrichtung hat, zeigt das Beispiel Bayern eindrucksvoll. So ist das Archiv der größten Universität, der LMU München, ebenso eine zentrale Einrichtung wie das einer mittleren Universität wie Augsburg oder dasjenige der kleinsten und jüngsten Universität Eichstätt. 


\section{DER ARBEITSKREIS DER BAYERISCHEN UNIVERSITÄTSARCHIVE}

Weitgehend unabhängig von Alter und Größe gehören die Universitätsarchive mit Blick auf ihre Ausstattung zu den kleineren Archiven. Daher stellt insbesondere die aufwändige Grundlagenarbeit, etwa die Erarbeitung von Aktenplänen, Aussonderungsrichtlinien oder Konzepten zur Archivierung digitaler Unterlagen eine besondere Herausforderung dar.

Viele Probleme und Herausforderungen betreffen das Archivwesen insgesamt, woraus sich ganz natürlich eine Zusammenarbeit der Universitätsarchive mit den größeren Archiven ergibt, insbesondere den Staatsarchiven, denen in solchen Fällen eine Vorreiterrolle zufällt. Jedoch unterscheiden sich die Universitätsarchive auch durch einige spezifische Besonderheiten und Unterschiede von den anderen Archiven. In den universitätsspezifischen Bereichen (etwa der Archivierung von wissenschaftlichen Forschungsprojekten oder von Prüfungsunterlagen) müssen entsprechende Lösungskonzepte eigenständig von den Hochschularchiven erarbeitet werden.

Für die Gründung des Arbeitskreises auf Landesebene sprachen dabei die gemeinsamen rechtlichen Grundlagen ${ }^{6}$, da das Archiv- und Hochschulrecht in die Zuständigkeit der Bundesländer fällt. Hinzu kommen praktische Faktoren wie die räumliche Nähe und eine für die Diskussion und Arbeit praktikable Mitgliederzahl, welche eine intensive Arbeit zulässt. Die Tätigkeit des Arbeitskreises findet ergänzend und in Zusammenarbeit mit derjenigen des VdA (Verband deutscher Archivarinnen und

6 BayArchivG und Bayerisches Hochschulgesetz vom 23. Mai 2006, erhältlich im Internet: http://by.juris.de/by/gesamt/HSchulG_BY_2006.htm (besucht am 7. März 2011). 
Archivare e.V.) und insbesondere der Fachgruppe 8 innerhalb des VdA statt.

Ausgehend von einer Initiative des Augsburger Universitätsarchivs (Dr. Lengger) konstituierte sich im Mai 2002 der Arbeitskreis der bayerischen Universitätsarchive, an dem zunächst sechs Universitäten ${ }^{7}$ beteiligt waren. An den Universitäten Augsburg, Erlangen und LMU München waren bereits in jüngerer Zeit Archive eingerichtet worden. Die Archive in Augsburg, Erlangen und an der LMU München waren kurz zuvor eingerichtet worden, die Archive an der TU München und in Würzburg befanden sich gerade im Aufbau, dasjenige in Bayreuth in der Planung.

\section{ZIELE}

Die Förderung und Unterstützung bei der Einrichtung von Archiven ergab sich als ein wichtiges Anliegen des Arbeitskreises. Inzwischen sind an fast allen bayerischen Universitäten Archive eingerichtet worden ${ }^{8}$. Eine der bedeutendsten Funktionen des Arbeitskreises ist die Bündelung und effiziente Nutzung des Fachund Detailwissens der einzelnen Mitglieder durch Kooperation der Universitätsarchive. Hierzu gehört ein intensiver Informationsaustausch, um auf Fragestellungen und Probleme aufmerksam zu machen. Verstärkt rückte auch die gezielte Fortbildung und Schulung in den Fokus des Arbeitskreises. Nach außen versteht

7 Universität Augsburg, Universität Bayreuth, Friedrich-AlexanderUniversität Erlangen-Nürnberg, Ludwig-Maximilians-Universität München, Technische Universität München, Julius-Maximilians-Universität Würzburg.

8 An der Universität Bayreuth fehlt ein vollwertiges Universitätsarchiv bisher noch. 
sich der Arbeitskreis als Interessenvertretung der Universitätsarchive und als Ansprechpartner in Fragen des universitären Archivwesens in Bayern.

\section{ARBEIT}

In halbjährlich stattfindenden, regelmäßigen Arbeitssitzungen sowie außerordentlichen Arbeitstreffen zu Spezialthemen hat sich der Arbeitskreis bisher mit den folgenden Themen beschäftigt:

- Archivierung von Massenakten (Prüfungsakten, Personalakten, Patientenakten) mit Richtlinien zur Ausdünnung von Massenakten

- Rechtliche Grundlagen der Universitätsarchive: die Satzung

- Vereinheitlichung von Benutzer- und Gebührenordnungen ${ }^{9}$

- Musteraktenplan

- Fristen- und Bewertungskatalog

- Personalpolitik in Universitätsarchiven

- Aufgaben und Probleme der Archive bei der Einführung eines Dokumentenmanagement- beziehungsweise Vorgangsbearbeitungssystems

- Langzeitarchivierung digitaler Unterlagen

- Klärung rechtlicher Fragen (insbesondere Datenschutz und Urheberrecht)

9 Hierbei wurden die Grundpositionen vereinheitlicht und somit die Benutzung transparenter gemacht. Eine Einheitsordnung für alle Hochschularchive wurde jedoch abgelehnt, um den Besonderheiten der einzelnen Universitäten gerecht werden zu können. 


\section{AUSBLICK}

Seit der Konstituierung des Arbeitskreises wurden inzwischen an fast allen bayerischen Universitäten Archive eingerichtet. Für die Zukunft stellt sich daher die Frage nach der Möglichkeit der Einbindung anderer wissenschaftlicher Archive, die auch in der Form projektbezogener Kooperationen stattfinden kann. So wurde jüngst das DEVA Projekt ${ }^{10}$ der Universität Bayreuth, die noch kein eigenes Universitätsarchiv besitzt, in den Arbeitskreis eingebunden. Die Herausforderungen der Archivierung digitaler Unterlagen werden zu einer Intensivierung der Kooperation mit der entsprechenden Arbeitsgruppe der Fachgruppe 8 des VdA führen. Der schnelle Austausch über aktuelle Probleme und Erfahrungen wird als eine der Stärken des Arbeitskreises aber weiterhin eine wichtige Rolle spielen.

10 Über das Projekt DEVA „Digitalisierung, Edition, Vernetzung in der Afrikawissenschaft" s.: http://www.ethnologie.uni-bayreuth.de/de/research/ DEVA/index.html (besucht am 5. März 2011). 


\title{
ARCHIWUM UNIWERSYTECKIE W WÜRZBURGU I JEGO MIEJSCE WŚRÓD BAWARSKICH ARCHIWÓW UNIWERSYTECKICH
}

\author{
(STRESZCZENIE)
}

Tematem artykułu jest pozycja Uniwersytetu w Würzburgu oraz jego archiwum wśród uczelni bawarskich i ich archiwów. Przedstawiona została również Grupa Robocza Bawarskich Archiwów Uniwersyteckich, jej cele oraz realizowane zadania. We wstępie mowa jest o historii założonego w 1402 roku Uniwersytetu w Würzburgu. Instytucja ta działała jedynie przez około 25 lat, wówczas to po raz ostatni wzmiankowana jest w źródłach. Uniwersytet ponownie powołał do życia książę-biskup Julius Echter z Mespelbrunn w 1582 roku. Od tego czasu - przez ponad 425 lat - placówka ta stawia czoła zmiennej historii, co znajduje swój wyraz również w zbiorach Archiwum Uniwersyteckiego.

Dzisiejsze Archiwum Uniwersyteckie utworzono w latach 2003-2008 na podstawie Bawarskiej Ustawy o Archiwach. Realizuje ono zadania w siedmiu obszarach, wśród których fundamentalne znaczenie mają: selekcja, udostępnianie zbiorów, ich utrzymanie, zarządzanie zbiorami i spuściznami oraz serwis dla użytkowników. Zasoby Archiwum obejmują 1500 metrów bieżących, przy czym warto zaznaczyć, że archiwalia sprzed 1700 roku w olbrzymiej części uległy zniszczeniu w wyniku zbombardowania Würzburga w dniu 16 marca 1945 roku.

W artykule przedstawiono również bawarskie uniwersytety oraz ich archiwa. Podobnie, jak w przypadku Uniwersytetu w Würzburgu, także uniwersytety w Monachium i w Erlangen zostały założone z inicjatywy lokalnych władców w czasach nowożytnych. W przeciwieństwie do Uniwersytetu Technicznego w Monachium utworzonego jako Szkoła Politechniczna, młodsze uniwersytety bawarskie w Regensburgu, Augsburgu, Bambergu i w Passawie powstały w wyniku przekształcenia w XX wieku istniejących w tych miejscowościach liceów. Początkowo 
w wyniku reorganizacji utworzono na ich bazie wyższe szkoły filozoficzno-teologiczne, które następnie przekształcono w uniwersytety. Wyjątkiem jest Bamberg - tamtejsze liceum powstało na bazie starego uniwersytetu. Uniwersytet w Bayreuth i Uniwersytet Wojskowy w Monachium zostały utworzone od podstaw, nie zaś w oparciu o istniejącą placówkę edykcyjną. Zwraca uwagę fakt, że cztery najstarsze uniwersytety cieszą się dziś największą liczbą studentów.

Ostatni rozdział artykułu poświęcony jest Grupie Roboczej Bawarskich Archiwów Uniwersyteckich. Stowarzyszenie to służy głównie wspieraniu i koordynacji współpracy archiwów uniwersyteckich z większymi archiwami, np. archiwami państwowymi, a także ze Związkiem Archiwistów Niemieckich (VdA). Grupa robocza powstała w maju 2002 roku z inicjatywy Archiwum Uniwersyteckiego w Augsburgu i skupiła w pierwszej fazie sześć uniwersytetów. Celem jej działalności jest dyskusja na temat warunków funkcjonowania archiwów uniwersyteckich oraz wspieranie pracy zatrudnionych w nich archiwistów, w szczególności wymiana doświadczeń, pogłębianie wiedzy fachowej, inicjowanie szkoleń.

Tłumaczenie/Übersetzt von Barbara Sapała 